\title{
Directly Detecting Sub-GeV Dark Matter with Electrons from Nuclear Scattering
}

\author{
Matthew J. Dolan, ${ }^{1, *}$ Felix Kahlhoefer, ${ }^{2, \dagger}$ and Christopher McCabe ${ }^{3, *}$ \\ ${ }^{1}$ ARC Centre of Excellence for Particle Physics at the Terascale, School of Physics, University of Melbourne, Victoria 3010, Australia \\ ${ }^{2}$ Institute for Theoretical Particle Physics and Cosmology (TTK), RWTH Aachen University, D-52056 Aachen, Germany \\ ${ }^{3}$ Department of Physics, King's College London, Strand, London, WC2R 2LS, United Kingdom
}

(Received 7 February 2018; revised manuscript received 18 July 2018; published 6 September 2018)

\begin{abstract}
Dark matter (DM) particles with mass in the sub-GeV range are an attractive alternative to heavier weakly interacting massive particles, but direct detection of such light particles is challenging. If, however, DM-nucleus scattering leads to ionization of the recoiling atom, the resulting electron may be detected even if the nuclear recoil is unobservable. We demonstrate that including this effect significantly enhances direct detection sensitivity to sub-GeV DM. Existing experiments set world-leading limits, and future experiments may probe the cross sections relevant for thermal freeze-out.
\end{abstract}

DOI: 10.1103/PhysRevLett.121.101801

Introduction.-Despite spectacular improvements in sensitivity over recent years, dark matter (DM) direct detection experiments have so far failed to observe conclusive evidence of a DM signal. While this may be interpreted as a failure of the paradigm of weakly interacting massive particles (WIMPs) [1-3], an alternative explanation is that WIMPs are lighter than usually assumed and that the energy they can deposit in a detector is below current experimental thresholds (see [4] for a recent review). This consideration has led to increasing interest in experiments with lower thresholds for nuclear recoils, such as CRESST [5-8], DAMIC [9], EDELWEISS [10], NEWS-G [11], or SuperCDMS [12], in experiments sensitive for electron recoils [13-16], or indeed in the development of completely novel types of detectors [17-33].

Direct detection experiments based on liquid xenon, on the other hand, are usually believed to be insensitive to nuclear recoils with sub-keV energy, corresponding to sub-GeV DM particles. This conclusion is based on the implicit assumption that the electron cloud of the recoiling atom instantly follows the nucleus, so that ionizations and excitations are only produced subsequently, as the recoiling atom collides with surrounding xenon atoms. The resulting primary scintillation ( $S 1)$ signal is then too small to be observable.

From neutron-nucleus scattering experiments, it is, however, known that the sudden acceleration of a nucleus after a collision leads to excitations and ionization of atomic electrons (see, e.g., [34-38]). This effect, illustrated in Fig. 1, can lead to energetic $\gamma$ rays and ionization electrons

Published by the American Physical Society under the terms of the Creative Commons Attribution 4.0 International license. Further distribution of this work must maintain attribution to the author(s) and the published article's title, journal citation, and DOI. Funded by SCOAP ${ }^{3}$. being produced from the primary interaction. The $S 1$ signal is then much larger and the sensitivity of liquid xenon detectors is significantly enhanced. The case of $\gamma$-ray emission was investigated in detail in [39]. Reference [40] furthermore showed that liquid xenon detectors such as LUX [41], XENON1T [42], or PandaX [43] can distinguish such events from electron recoils, which constitute the main background.

Recently, Ref. [44] pointed out that the probability to ionize a recoiling atom is in fact substantially larger than the probability to obtain a $\gamma$ ray. This effect has been named the "Migdal effect" in the DM literature [45-47], as the calculation makes use of the Migdal approximation [48] that the electron cloud of the atom does not change during the nuclear recoil induced by the DM interaction (see Fig. 1). In the frame of the nucleus, this results in a simultaneous boost for all electrons, which can lead to excitation or ionization of electrons.

In this Letter, we further explore the formalism developed in [44] and apply it to the case of liquid xenon detectors. We find that the sensitivity of this type of experiment in the
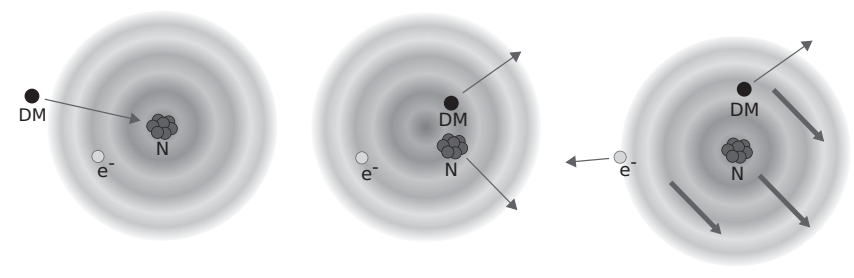

FIG. 1. Illustration of electron emission from nuclear recoils. If a DM particle scatters off a nucleus (left), we can assume that immediately after the collision the nucleus moves relative to the surrounding electron cloud (middle). The electrons eventually catch up with the nucleus, but individual electrons may be left behind and are emitted, leading to ionization of the recoiling atom (right). 
sub-GeV mass range is significantly enhanced. By reinterpreting existing data from the LUX and XENON1T experiments, we obtain the strongest limit on DM with mass between $\sim 0.1$ and $0.5 \mathrm{GeV}$ and comparable limits to CRESST-III [8] between $\sim 0.5$ and $1 \mathrm{GeV}$. A second central observation of our Letter is that, in scenarios where the DM couples with equal strength to electrons and protons (as in the case of interactions mediated by a dark photon with kinetic mixing [49,50]), experiments may be more sensitive to ionization electrons resulting from nuclear recoils than from electron recoils. The search strategy considered in this Letter can therefore probe unexplored parameter regions of dark photon models and future experiments may be sensitive to parameter space compatible with thermal freeze-out.

We begin by reviewing the central aspects of the Migdal effect and the relevant formulas from the literature. Next, we discuss the resulting signatures in liquid xenon detectors, considering the LUX experiment for concreteness. Finally, we present the resulting bounds on the parameter space of two interesting models of light DM.

Ionization electrons from nuclear recoils.-In this section, we summarize the key results from [44] needed for calculating the signatures of light DM scattering on nuclei in direct detection experiments. The differential event rate for DM-nucleus scattering $R_{\mathrm{nr}}$ with respect to the nuclear recoil energy $E_{R}$ and the DM velocity $v$ is given by

$$
\frac{d^{2} R_{\mathrm{nr}}}{d E_{R} d v}=\frac{\rho \sigma_{N}}{2 \mu_{N}^{2} m_{\mathrm{DM}}} \frac{f(v)}{v},
$$

where $\rho$ denotes the local DM density, $\sigma_{N}$ is the DM-nucleus scattering cross section, $m_{\mathrm{DM}}$ is the DM mass, $\mu_{N}=$ $m_{N} m_{\mathrm{DM}} /\left(m_{N}+m_{\mathrm{DM}}\right)$ is the DM-nucleus reduced mass, and $f(v)=\int v^{2} f(\mathbf{v}) d \Omega_{v}$ is the DM speed distribution in the laboratory frame [51]. (We have absorbed the coherent enhancement factor into our definition of $\sigma_{N}$.) We neglect nuclear form factors since we are only interested in small momentum transfers. The differential event rate for a nuclear recoil of energy $E_{R}$ to be accompanied by an ionization electron with energy $E_{e}$ is

$$
\frac{d^{3} R_{\mathrm{ion}}}{d E_{R} d E_{e} d v}=\frac{d^{2} R_{\mathrm{nr}}}{d E_{R} d v} \times\left|Z_{\mathrm{ion}}\left(E_{R}, E_{e}\right)\right|^{2},
$$

where the transition rate is given by

$$
\left|Z_{\text {ion }}\left(E_{R}, E_{e}\right)\right|^{2}=\sum_{n l} \frac{1}{2 \pi} \frac{d p_{q_{e}}^{c}\left(n l \rightarrow E_{e}\right)}{d E_{e}} .
$$

In this expression, $n$ and $l$ denote the initial quantum numbers of the electron being emitted, $q_{e}=m_{e} \sqrt{2 E_{R} / m_{N}}$ is the momentum of each electron in the rest frame of the nucleus immediately after the scattering process, and $p_{q_{e}}^{c}\left(n l \rightarrow E_{e}\right)$ quantifies the probability to emit an electron with final kinetic energy $E_{e}$. We can make the dependence of $p_{q_{e}}^{c}\left(n l \rightarrow E_{e}\right)$ on $q_{e}$ explicit by writing

$$
p_{q_{e}}^{c}\left(n l \rightarrow E_{e}\right)=\left(\frac{q_{e}}{v_{\mathrm{ref}} m_{e}}\right) p_{v_{\mathrm{ref}}}^{c}\left(n l \rightarrow E_{e}\right),
$$

where $v_{\text {ref }}$ is a fixed reference velocity. The functions $p_{v_{\text {ref }}}^{c}\left(n l \rightarrow E_{e}\right)$ depend on the target material under consideration. We use the functions from Ref. [44], which have been calculated taking $v_{\text {ref }}=10^{-3}$.

If the emitted electron comes from an inner orbital, the remaining ion will be in an excited state. To return to the ground state, further electronic energy will be released in the form of photons or additional electrons. (In contrast, the probability to obtain double ionization from the Migdal effect itself is exceedingly small $[52,53]$.) The total electronic energy deposited in the detector is hence approximately given by $E_{\mathrm{EM}}=E_{e}+E_{n l}$, where $E_{n l}$ is the (positive) binding energy of the electron before emission.

We integrate Eq. (2) over the nuclear recoil energy and the DM velocity to calculate the energy spectrum, including only those combinations of $E_{R}, E_{\mathrm{EM}}$, and $v$ that satisfy energy and momentum conservation. The resulting calculation is identical to the case of inelastic DM [54], with the DM mass splitting $\Delta m$ being replaced by the total electronic energy $E_{\mathrm{EM}}$. (We neglect the difference in mass between the original atom and the recoiling excited state.) We find

$$
v_{\min }=\sqrt{\frac{m_{N} E_{R}}{2 \mu^{2}}}+\frac{E_{\mathrm{EM}}}{\sqrt{2 m_{N} E_{R}}} .
$$

The maximum electronic and nuclear recoil energy for a given DM mass are given by

$$
E_{R, \max }=\frac{2 \mu_{N}^{2} v_{\max }^{2}}{m_{N}}, \quad E_{\mathrm{EM}, \max }=\frac{\mu_{N} v_{\max }^{2}}{2} .
$$

For $v_{\max } \approx 800 \mathrm{~km} / \mathrm{s}, m_{\mathrm{DM}} \ll m_{N}$ (and hence $\mu_{N} \approx m_{\mathrm{DM}}$ ), we generically find $E_{\mathrm{EM}, \max } \gg E_{R, \max }$. For concreteness, for $m_{\mathrm{DM}}=0.5 \mathrm{GeV}$ and $m_{N}=120 \mathrm{GeV}$ (the approximate xenon atom mass), we find $E_{R, \max } \approx 0.03 \mathrm{keV}$, while $E_{\mathrm{EM}, \max } \approx 1.8 \mathrm{keV}$. The electronic energy is therefore much easier to detect than the nuclear recoil energy.

Sensitivity of liquid xenon detectors.-Having obtained the relevant formulas for the distribution of electronic and nuclear recoil energy at the interaction point where the DM-nucleus scattering occurs, we now convert these energies into observables accessible for direct detection experiments. The focus of this discussion will be on liquid xenon detectors, but we note that the dominance of the electronic energy $E_{\mathrm{EM}}$ resulting from the Migdal effect is not limited to xenon. These detectors convert the atomic excitations and ionizations at the interaction point into a primary $(S 1)$ and a secondary $(S 2)$ scintillation signal [55]. A specific detector can be characterized by two functions: $\operatorname{PDF}\left(S 1, S 2 \mid E_{R}, E_{\mathrm{EM}}\right)$ quantifies the probability 
to obtain specific $S 1$ and $S 2$ values for given $E_{R}$ and $E_{\mathrm{EM}}$, and $\epsilon(S 1, S 2)$ quantifies the probability that a signal with given $S 1$ and $S 2$ will be detected and will satisfy all selection cuts. Using these two functions, we can write

$$
\begin{aligned}
\frac{d^{2} R}{d S 1 d S 2}= & \epsilon(S 1, S 2) \int d E_{R} d E_{\mathrm{EM}} \frac{d^{2} R}{d E_{R} d E_{\mathrm{EM}}} \\
& \times \operatorname{PDF}\left(S 1, S 2 \mid E_{R}, E_{\mathrm{EM}}\right),
\end{aligned}
$$

where we have now expressed the differential event rate from Eq. (2) in terms of $E_{\mathrm{EM}}$.

For sub-GeV DM particles, nuclear recoil energies are below $\mathcal{O}(0.1) \mathrm{keV}$. The scintillation and ionization yield for such small energies have not yet been measured in liquid xenon, but theoretical arguments predict the resulting signals to be very small [56]. We conservatively neglect this contribution and assume that only electronic energy contributes to the $S 1$ and $S 2$ signals, such that $\operatorname{PDF}\left(S 1, S 2 \mid E_{R}, E_{\mathrm{EM}}\right)=\operatorname{PDF}\left(S 1, S 2 \mid E_{\mathrm{EM}}\right)$ and the integration over $E_{R}$ in Eq. (7) can be immediately performed.

References [40,57] and the Supplemental Material [58] discuss how we determine $\operatorname{PDF}\left(S 1, S 2 \mid E_{\mathrm{EM}}\right)$ and $\epsilon(S 1, S 2)$ for liquid xenon experiments using a Monte Carlo simulation of the detector based on the Noble Element Simulation Technique [62-64]. For given $E_{\mathrm{EM}}$, the mean $S 1$ and $S 2$ signals can be written as $S 1=g_{1} L_{y} E_{\mathrm{EM}}$ and $S 2=g_{2} Q_{y} E_{\mathrm{EM}}$, respectively, where $g_{1,2}$ are detectordependent gain factors and $L_{y}$ and $Q_{y}$ are properties of liquid xenon determined from calibration data [65-69]. The Monte Carlo simulation then determines the probability for fluctuations in the number of excited and ionized atoms produced initially, recombination fluctuations, finite extraction, and detection efficiencies.

As pointed out in [40], these fluctuations play a crucial role for the sensitivity of liquid xenon detectors to sub-GeV DM particles, because the mean $S 1$ signal expected from the scattering of light DM particles lies below the detection threshold of typical detectors. Thus, the signal can only be observed in the case of an upward fluctuation of the $S 1$ signal. Moreover, events with unusually large $S 1$ signal have the advantage that they do not look like typical electronic recoils. Instead, they look more similar to nuclear recoils, which have a smaller ratio of $S 2 / S 1$ than electron recoils. This feature makes it possible to distinguish between the expected signal and the main sources of backgrounds.

We focus on the LUX [41,70] and XENON1T [42,71] experiments, and following [40], implement two different approaches to estimate the sensitivity to sub-GeV DM, adopting the astrophysical parameters used in [40] for our analysis. For the cut-and-count (CC) approach, we determine the region in $S 1-S 2$ space that contains $90 \%$ of the DM events passing all cuts and count the number of observed events in this region. A limit can then be set by assuming all these events to be signal events and calculating a Poisson upper bound on the expected number of events (at $90 \%$ confidence level). A more powerful approach is the profile likelihood method (PLR), which takes into account the likelihood for signal and background at each observed event $[72,73]$. In contrast to the CC approach, the PLR method requires a background model. For LUX, our model consists of two components: a component flat in energy from electronic recoils and a component from the decays of ${ }^{127} \mathrm{Xe}$. Further details are given in [40]. For XENON1T, modeling the surface background is beyond the scope of this Letter, and so we only show results for a CC approach. With this approach, we obtain a stronger bound from the first science run (SR0) than from the second science run (SR1) due to the smaller number of background events. We expect that a PLR analysis of SR1 would further improve the XENON1T exclusion limit.

We also estimate the expected sensitivity of the LZ experiment [74], and note that a similar sensitivity should be expected with XENONnT [75]. We show results assuming 1000 days of data taking and a 5.6 metric ton target volume using the detector parameters sets discussed in [76].

Results.-We present the results of our analysis for DM particles interacting with nuclei via two different types of mediators: scalars and vectors. The difference between these two cases is how the mediator couples to standard model (SM) particles [77]. For a scalar mediator, these couplings generically arise from mixing with the SM Higgs boson, so that the mediator couples to SM particles proportional to their mass. In particular, the DM-nucleus cross section for nuclei with mass number $A$ is enhanced by a coherence factor $\sigma_{N}=A^{2} \sigma_{p} \mu_{N}^{2} / \mu_{p}^{2}$, where $\sigma_{p}$ and $\mu_{p}$ are the DM-proton cross section and reduced mass, respectively. Furthermore, couplings to electrons are negligible. Models with sub-GeV DM particles and (light) scalar mediators have, e.g., been considered recently in the context of self-interacting DM $[78,79]$.

For vector mediators, couplings to SM particles arise from kinetic mixing with the photon. As a result, couplings to SM particles are expected to be proportional to their electromagnetic charge, leading to a $Z^{2}$ enhancement for scattering on nuclei with charge number $Z$, i.e., $\sigma_{N}=$ $Z^{2} \sigma_{p} \mu_{N}^{2} / \mu_{p}^{2}$, and comparable couplings to protons and electrons. These so-called dark photon models have been studied extensively in the literature [15,16,80-85].

We emphasize that, even if the couplings of DM to protons and electrons are comparable, the corresponding scattering cross sections are not, because they are proportional to the reduced mass squared [13]. For sub-GeV DM particles, one finds $\mu_{p} \approx m_{\mathrm{DM}}$ and $\mu_{e} \approx m_{e}$, so scattering on nucleons is enhanced by a factor $m_{\mathrm{DM}}^{2} / m_{e}^{2}$. For heavy atoms, the coherence factor for the nucleus leads to an additional enhancement in spite of the larger number density of electrons. Thus, the probability for DM particles in the mass range $0.1-1 \mathrm{GeV}$ to scatter on nuclei is many orders of magnitude larger than the probability to scatter on electrons. Most of these scattering processes will be 
unobserved, but even a small fraction of events with ionization electrons are sufficient to obtain strong constraints.

Our results are summarized in Fig. 2, focusing on the mass range $0.1 \leq m_{\mathrm{DM}} \leq 1 \mathrm{GeV}$. The upper panel shows the case of a scalar mediator (Higgs mixing), and the lower panel shows the case of a vector mediator (kinetic mixing). An additional assumption in both plots is that the mediator is sufficiently heavy that the scattering can be described by contact interactions, which is the case for $m_{\text {med }} \gtrsim 10 \mathrm{MeV}$ [79]. No further assumptions are needed to compare the constraints from different direct detection experiments looking for nuclear or electron recoils. (We do not show constraints from hidden photon searches at BABAR [82], which require the assumption of a specific ratio between the DM mass and the mediator mass.) Projected sensitivities are taken from [12] for SuperCDMS-Ge, from [79] for CRESST-III, and from [4] for SENSEI, DAMIC, and SuperCDMS-Si.

We find that, in both of the cases considered, the sensitivity of current liquid xenon detectors for sub-GeV DM can compete with other existing and proposed strategies. In the case of a scalar mediator, only experiments sensitive to nuclear recoils give relevant constraints. We find that XENON1T gives the world-leading exclusion limit across the entire mass range under consideration. In particular, we find XENON1T to be more constraining than the first analysis of CRESST-III [8] even for $m_{\mathrm{DM}} \gtrsim 0.5 \mathrm{GeV}$. For lower DM masses, we significantly improve the LUX bound obtained from $\gamma$ rays emitted in nuclear scattering processes [40] and from the CRESST 2017 surface run [7].

In the case of vector mediators, constraints from LUX and XENON1T are slightly weakened relative to the ones from CRESST due to a smaller difference in the enhancement factors. In addition, there are now strong constraints from searches for electron scattering in XENON100 [16,86]. Nevertheless, we observe that searches for electrons emitted for nuclear recoils in liquid xenon detectors set competitive bounds for DM masses around 200-400 MeV. LZ can significantly improve upon these bounds in the future and provide complementary constraints to alternative electronrecoil strategies proposed to search for sub-GeV DM [4].

To put our results into context, it is instructive to compare the sensitivity of direct detection experiments to the parameter regions where the DM particle can be a thermal relic that obtains its abundance via the freeze-out mechanism. Such a comparison is necessarily model dependent, but the number of possibilities is limited by strong constraints on sub-GeV WIMPs $[87,88]$. Here we focus on one viable scenario, namely, a vector mediator and complex scalar DM [15]. If the mediator mass is sufficiently large, $m_{\text {med }}>2 m_{\mathrm{DM}}$, the DM relic abundance probes the same combination of parameters as direct detection experiments. In other words, for each value of $m_{\mathrm{DM}}$, there is a unique value of $\sigma_{p}$ corresponding to the observed relic abundance [89]. The scattering cross sections obtained in this way are indicated by the gray band
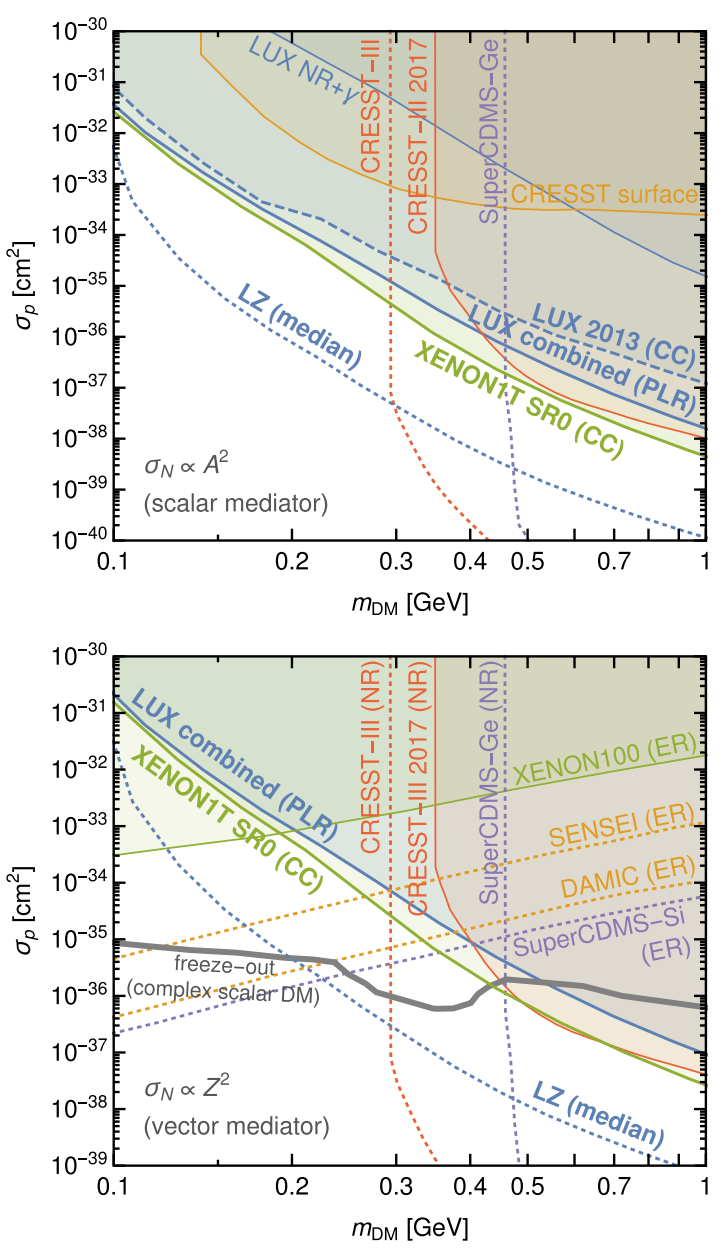

FIG. 2. Exclusion limits (solid lines) and projected sensitivities (dotted lines) for sub-GeV DM. The bounds resulting from our analysis of electron emission after nuclear recoils are shown in blue for LUX (using two different statistical methods) and in green for XENON1T. (Upper) The case of a scalar mediator (couplings proportional to mass). (Lower) The case of a vector mediator (couplings proportional to charge). In the latter case, there are constraints both from experiments looking for nuclear recoils (NR) and from experiments looking for electron recoils (ER). The bounds from the CRESST surface run, LUX NR $+\gamma$ and LUX 2013 (CC) have been omitted in the lower panel for clarity. We also show the parameter combinations that yield the observed DM relic abundance for one specific model (complex scalar DM). See text for details.

in the lower panel of Fig. 2. We observe that current bounds exclude the simplest realization of thermal freeze-out in the model that we consider for $m_{\mathrm{DM}}>450 \mathrm{MeV}$, while the next generation of direct detection experiments is expected to probe the relevant cross sections across the entire mass range of interest.

Conclusions.-The sub-GeV mass range represents a new frontier in the search for particle DM. While direct detection experiments are often considered insensitive to nuclear recoils induced by scattering of light DM particles, we have shown that electrons emitted from recoiling atoms 
significantly boost the signal, leading to an enhanced sensitivity for, and new bounds on, the DM-nucleus scattering cross section. In liquid xenon detectors, the sensitivity to these signals is further enhanced by the possibility of upward fluctuations in the $S 1$ signal, leading to the possibility to distinguish between signal and background.

In the present Letter, we have focused on the LUX experiment as an example of existing technology and the LZ experiment as illustration of the power of nextgeneration experiments. Nevertheless, the same physics will be relevant for other direct detection experiments sensitive to electronic recoils. Moreover, our results may also be applied to coherent neutrino-nucleus scattering $[44,90]$ and to the interpretation of calibration data based on neutron-nucleus scattering $[91,92]$.

In conclusion, we emphasize that direct detection experiments are only just beginning to probe the interesting parameter space for sub-GeV WIMPs. Significant improvements of sensitivity are required in order to probe the cross sections favored by the freeze-out mechanism. To achieve this goal, we need both a dedicated effort to build new types of direct detection experiments with very low thresholds and further theoretical developments to better understand the ways in which DM particles can lead to observable signals in conventional direct detection experiments.

We thank Josef Pradler and Tien-Tien Yu for discussions and Florian Reindl for correspondence. M. J. D. is supported by the Australian Research Council and thanks King's College London. F. K. is supported by the DFG Emmy Noether Grant No. KA 4662/1-1. C. M. is supported by the Science and Technology Facilities Council (STFC) Grant No. ST/N004663/1.

*dolan@unimelb.edu.au

†kahlhoefer@physik.rwth-aachen.de

*christopher.mccabe@kcl.ac.uk

[1] M. Duerr, F. Kahlhoefer, K. Schmidt-Hoberg, T. Schwetz, and S. Vogl, J. High Energy Phys. 09 (2016) 042.

[2] M. Escudero, A. Berlin, D. Hooper, and M.-X. Lin, J. Cosmol. Astropart. Phys. 12 (2016) 029.

[3] G. Arcadi, M. Dutra, P. Ghosh, M. Lindner, Y. Mambrini, M. Pierre, S. Profumo, and F. S. Queiroz, Eur. Phys. J. C 78, 203 (2018).

[4] M. Battaglieri et al., arXiv:1707.04591.

[5] G. Angloher et al. (CRESST Collaboration), Eur. Phys. J. C 76, 25 (2016).

[6] G. Angloher et al. (CRESST Collaboration), arXiv:1503 .08065 .

[7] G. Angloher et al. (CRESST Collaboration), Eur. Phys. J. C 77, 637 (2017).

[8] F. Petricca et al. (CRESST Collaboration), arXiv:1711 .07692 .

[9] A. Aguilar-Arevalo et al. (DAMIC Collaboration), Phys. Rev. D 94, 082006 (2016).
[10] Q. Arnaud et al. (EDELWEISS Collaboration), Phys. Rev. D 97, 022003 (2018).

[11] Q. Arnaud et al., Astropart. Phys. 97, 54 (2018).

[12] R. Agnese et al. (SuperCDMS Collaboration), Phys. Rev. D 95, 082002 (2017).

[13] R. Essig, J. Mardon, and T. Volansky, Phys. Rev. D 85, 076007 (2012).

[14] P. W. Graham, D. E. Kaplan, S. Rajendran, and M. T. Walters, Phys. Dark Universe 1, 32 (2012).

[15] R. Essig, M. Fernandez-Serra, J. Mardon, A. Soto, T. Volansky, and T.-T. Yu, J. High Energy Phys. 05 (2016) 046.

[16] R. Essig, T. Volansky, and T.-T. Yu, Phys. Rev. D 96, 043017 (2017).

[17] W. Guo and D. N. McKinsey, Phys. Rev. D 87, 115001 (2013).

[18] Y. Hochberg, Y. Zhao, and K. M. Zurek, Phys. Rev. Lett. 116, 011301 (2016).

[19] Y. Hochberg, M. Pyle, Y. Zhao, and K. M. Zurek, J. High Energy Phys. 08 (2016) 057.

[20] Y. Hochberg, T. Lin, and K. M. Zurek, Phys. Rev. D 94, 015019 (2016).

[21] K. Schutz and K. M. Zurek, Phys. Rev. Lett. 117, 121302 (2016).

[22] F. W. Carter, S. A. Hertel, M. J. Rooks, P. V. E. McClintock, D. N. McKinsey, and D. E. Prober, J. Low Temp. Phys. 186, 183 (2017).

[23] Y. Hochberg, Y. Kahn, M. Lisanti, C. G. Tully, and K. M. Zurek, Phys. Lett. B 772, 239 (2017).

[24] S. Derenzo, R. Essig, A. Massari, A. Soto, and T.-T. Yu, Phys. Rev. D 96, 016026 (2017).

[25] Y. Hochberg, T. Lin, and K. M. Zurek, Phys. Rev. D 95, 023013 (2017).

[26] R. Essig, J. Mardon, O. Slone, and T. Volansky, Phys. Rev. D 95, 056011 (2017).

[27] S. Knapen, T. Lin, and K. M. Zurek, Phys. Rev. D 95, 056019 (2017).

[28] P. C. Bunting, G. Gratta, T. Melia, and S. Rajendran, Phys. Rev. D 95, 095001 (2017).

[29] R. Budnik, O. Chesnovsky, O. Slone, and T. Volansky, Phys. Lett. B 782, 242 (2018).

[30] J. Tiffenberg, M. Sofo-Haro, A. Drlica-Wagner, R. Essig, Y. Guardincerri, S. Holland, T. Volansky, and T.-T. Yu, Phys. Rev. Lett. 119, 131802 (2017).

[31] H. J. Maris, G. M. Seidel, and D. Stein, Phys. Rev. Lett. 119, 181303 (2017).

[32] Y. Hochberg, Y. Kahn, M. Lisanti, K. M. Zurek, A. Grushin, R. Ilan, S. M. Griffin, Z.-F. Liu, and S. F. Weber, Phys. Rev. D 97, 015004 (2018).

[33] S. Fichet, Phys. Rev. Lett. 120, 131801 (2018).

[34] T. W. Ruijgrok, B. R. A. Nijboer, and M. R. Hoare, Physica (Amsterdam) 120A, 537 (1983).

[35] L. Vegh, J. Phys. B 16, 4175 (1983).

[36] G. Baur, F. Rosel, and D. Trautmann, J. Phys. B 16, L419 (1983).

[37] M. S. Pindzola, T. G. Lee, S. A. Abdel-Naby, F. Robicheaux, J. Colgan, and M. F. Ciappina, J. Phys. B 47, 195202 (2014).

[38] P. Sharma, Nucl. Phys. A968, 326 (2017).

[39] C. Kouvaris and J. Pradler, Phys. Rev. Lett. 118, 031803 (2017). 
[40] C. McCabe, Phys. Rev. D 96, 043010 (2017).

[41] D. S. Akerib et al. (LUX Collaboration), Phys. Rev. Lett. 118, 021303 (2017).

[42] E. Aprile et al. (XENON Collaboration), Phys. Rev. Lett. 119, 181301 (2017).

[43] X. Cui et al. (PandaX-II Collaboration), Phys. Rev. Lett. 119, 181302 (2017).

[44] M. Ibe, W. Nakano, Y. Shoji, and K. Suzuki, J. High Energy Phys. 03 (2018) 194.

[45] J. D. Vergados and H. Ejiri, Phys. Lett. B 606, 313 (2005).

[46] C. C. Moustakidis, J. D. Vergados, and H. Ejiri, Nucl. Phys. B727, 406 (2005).

[47] R. Bernabei et al., Int. J. Mod. Phys. A 22, 3155 (2007).

[48] L. D. Landau and E. M. Lifshitz, Quantum Mechanics: NonRelativistic Theory (Pergamon Press, New York, 1965).

[49] R. Foot, Int. J. Mod. Phys. D 13, 2161 (2004).

[50] J. L. Feng, M. Kaplinghat, H. Tu, and H.-B. Yu, J. Cosmol. Astropart. Phys. 07 (2009) 004.

[51] C. McCabe, J. Cosmol. Astropart. Phys. 02 (2014) 027.

[52] J. Feist, S. Nagele, R. Pazourek, E. Persson, B. I. Schneider, L. A. Collins, and J. Burgdörfer, Phys. Rev. A 77, 043420 (2008).

[53] M. Liertzer, J. Feist, S. Nagele, and J. Burgdörfer, Phys. Rev. Lett. 109, 013201 (2012).

[54] D. Smith and N. Weiner, Phys. Rev. D 64, 043502 (2001).

[55] V. Chepel and H. Araujo, J. Instrum. 8, R04001 (2013).

[56] P. Sorensen, Phys. Rev. D 91, 083509 (2015).

[57] C. McCabe, J. Cosmol. Astropart. Phys. 05 (2016) 033.

[58] See the Supplemental Material at http://link.aps.org/ supplemental/10.1103/PhysRevLett.121.101801 for $S 1-S 2$ profiles, electron efficiency and $Q_{y}$ curves that we used in our simulations, which includes Refs. [59-61].

[59] J. Balajthy, APS April Meeting (2018), https://meetings.aps .org/Meeting/APR18/Session/J09.3.

[60] D. S. Akerib et al. (LUX Collaboration), Phys. Rev. D 97, 102008 (2018).

[61] J. Aalbers, Ph.D. thesis, University of Amsterdam (2018).

[62] M. Szydagis, N. Barry, K. Kazkaz, J. Mock, D. Stolp, M. Sweany, M. Tripathi, S. Uvarov, N. Walsh, and M. Woods, J. Instrum. 6, P10002 (2011).

[63] M. Szydagis, A. Fyhrie, D. Thorngren, and M. Tripathi, J. Instrum. 8, C10003 (2013).

[64] B. Lenardo, K. Kazkaz, A. Manalaysay, J. Mock, M. Szydagis, and M. Tripathi, IEEE Trans. Nucl. Sci. 62, 3387 (2015).

[65] D. S. Akerib et al. (LUX Collaboration), Phys. Rev. D 93, 072009 (2016).

[66] L. W. Goetzke, E. Aprile, M. Anthony, G. Plante, and M. Weber, Phys. Rev. D 96, 103007 (2017).

[67] E. M. Boulton et al., J. Instrum. 12, P08004 (2017).
[68] D. S. Akerib et al. (LUX Collaboration), Phys. Rev. D 96, 112011 (2017).

[69] E. Aprile et al. (XENON Collaboration), Phys. Rev. D 97, 092007 (2018).

[70] D. S. Akerib et al. (LUX Collaboration), Phys. Rev. Lett. 116, 161301 (2016).

[71] E. Aprile et al. (XENON Collaboration), arXiv:1805.12562 [Phys. Rev. Lett. (to be published)].

[72] R. J. Barlow, Nucl. Instrum. Methods Phys. Res., Sect. A 297, 496 (1990).

[73] G. Cowan, K. Cranmer, E. Gross, and O. Vitells, Eur. Phys. J. C 71, 1 (2011); 73, 2501(E) (2013).

[74] B. J. Mount et al., arXiv:1703.09144.

[75] E. Aprile et al. (XENON Collaboration), J. Cosmol. Astropart. Phys. 04 (2016) 027.

[76] D. S. Akerib et al. (LUX-ZEPLIN Collaboration), arXiv: 1802.06039.

[77] M. Kaplinghat, S. Tulin, and H.-B. Yu, Phys. Rev. D 89, 035009 (2014).

[78] F. Kahlhoefer, K. Schmidt-Hoberg, and S. Wild, J. Cosmol. Astropart. Phys. 08 (2017) 003.

[79] F. Kahlhoefer, S. Kulkarni, and S. Wild, J. Cosmol. Astropart. Phys. 11 (2017) 016.

[80] H. An, M. Pospelov, J. Pradler, and A. Ritz, Phys. Lett. B 747, 331 (2015).

[81] R. Essig, J. Mardon, M. Papucci, T. Volansky, and Y.-M. Zhong, J. High Energy Phys. 11 (2013) 167.

[82] J. P. Lees et al. (BABAR Collaboration), Phys. Rev. Lett. 119, 131804 (2017).

[83] S. Andreas, C. Niebuhr, and A. Ringwald, Phys. Rev. D 86, 095019 (2012).

[84] E. Izaguirre, G. Krnjaic, P. Schuster, and N. Toro, Phys. Rev. D 88, 114015 (2013).

[85] B. Batell, R. Essig, and Z. Surujon, Phys. Rev. Lett. 113, 171802 (2014).

[86] E. Aprile et al. (XENON Collaboration), Phys. Rev. D 94, 092001 (2016); 95, 059901(E) (2017).

[87] C. Boehm, M. J. Dolan, and C. McCabe, J. Cosmol. Astropart. Phys. 08 (2013) 041.

[88] S. Knapen, T. Lin, and K. M. Zurek, Phys. Rev. D 96, 115021 (2017).

[89] P. A. R. Ade et al. (Planck Collaboration), Astron. Astrophys. 594, A13 (2016).

[90] D. Akimov et al. (COHERENT Collaboration), Science 357, 1123 (2017).

[91] J. R. Verbus et al., Nucl. Instrum. Methods Phys. Res., Sect. A 851, 68 (2017).

[92] P. S. Barbeau, J. I. Collar, and P. M. Whaley, Nucl. Instrum. Methods Phys. Res., Sect. A 574, 385 (2007). 